\title{
Minimal invasive puncture and drainage versus endoscopic surgery for spontaneous intracerebral hemorrhage in basal ganglia
}

This article was published in the following Dove Press journal:

Neuropsychiatric Disease and Treatment

25 January 2017

Number of times this article has been viewed

\author{
Zhihong $\mathrm{Li}^{1, *}$ \\ Yuqian $\mathrm{Li}^{1, *}$ \\ Feifei $\mathrm{Xu}^{2, *}$ \\ Xi Zhang ${ }^{3}$ \\ Qiang Tian ${ }^{4}$ \\ Lihong $\mathrm{Li}^{\prime}$ \\ 'Department of Neurosurgery, \\ Tangdu Hospital, ${ }^{2}$ Department of \\ Foreign Languages, ${ }^{3}$ Department of \\ Biomedical Engineering, ${ }^{4}$ Department \\ of Radiology, Tangdu Hospital, The \\ Fourth Military Medical University, \\ Xi'an, Shaanxi Province, People's \\ Republic of China \\ *These authors contributed equally \\ to this work
}

\begin{abstract}
Two prevalent therapies for the treatment of spontaneous intracerebral hemorrhage (ICH) in basal ganglia are, minimally invasive puncture and drainage (MIPD), and endoscopic surgery (ES). Because both surgical techniques are of a minimally invasive nature, they have attracted greater attention in recent years. However, evidence comparing the curative effect of MIPD and ES has been uncertain. The indication for MIPD or ES has been uncertain till now. In the present study, 112 patients with spontaneous ICH in basal ganglia who received MIPD or ES were reviewed retrospectively. Baseline parameters prior to the operation, evacuation rate (ER), perihematoma edema, postoperative complications, and rebleeding incidences were collected. Moreover, 1-year postictus, the long-term functional outcomes of patients with regard to hematoma volume (HV) or Glasgow Coma Scale (GCS) score were judged, respectively, by the case fatality, Glasgow Outcome Scale (GOS), Barthel Index (BI), and modified Rankin Scale (mRS). The ES group had a higher ER than the MIPD group on postoperative day 1 . The MIPD group had fewer adverse outcomes, which included less perihematoma edema, anesthetic time, and blood loss, than the ES group. The functional outcomes represented by GOS, BI, and $\mathrm{mRS}$ were better in the MIPD group than in the ES group for patients with HV 30-60 mL or GCS score 9-14. These results indicate that ES is more effective in evacuating hematoma in basal ganglia, while MIPD is less invasive than ES. Patients with HV 30-60 mL or GCS score 9-14 may benefit more from the MIPD procedure than from ES.
\end{abstract}

Keywords: intracerebral hemorrhage, minimal invasive, endoscopy, puncture, basal ganglia

\section{Abbreviations}

BI, Barthel Index; CT, computed tomography; DM, diabetes mellitus; ER, evacuation rate; ES, endoscopic surgery; HV, hematoma volume; ICH, intracerebral hemorrhage; IVH, intraventricular hemorrhage; GCS, Glasgow Coma Scale; GOS, Glasgow Outcome Scale; MIPD, minimally invasive puncture and drainage; MISTIE, Minimally Invasive Surgery Plus recombinant tissue-type plasminogen activator for ICH Evacuation; mRS, modified Rankin Scale; PHE, perihematomal edema; STICH, Surgical Trial in Intracerebral Hemorrhage.

\section{Introduction}

Spontaneous ICH is one of the most devastating forms of cerebrovascular disease with extremely high mortality ${ }^{1}$ and disability rate. $^{2}$ Among the several common regions of $\mathrm{ICH}$, the basal ganglia are most frequently affected. ICH remains the cerebrovascular disease without definitive treatments. The role of surgical treatments for ICH continues to be controversial and needs further investigation. Surgical treatments available
Department of Neurosurgery, Tangdu Hospital, The Fourth Military Medical University, I Xinyi Road, Baqiao District, Xi'an 710039, Shaanxi Province, People's Republic of China

Tel +8602984778359

Email lihongliaaa@I26.com BY NC and incorporate the Creative Commons Attribution - Non Commercial (unported, v3.0) License (http://creativecommons.org/licenses/by-nc/3.0/). By accessing the work you
hereby accept the Terms. Non-commercial uses of the work are permitted without any further permission from Dove Medical Press Limited, provided the work is properly attributed. For permission for commercial use of this work, please see paragraphs 4.2 and 5 of our Terms (https://www.dovepress.com/terms.php). 
for ICH can be divided primarily into the following three types: hematoma evacuation via craniotomy, MIPD, and ES. Craniotomy has ever been the conventional choice for $\mathrm{ICH}$ for many years. However, its role in ICH is uncertain till now. The randomized prospective trials, STICH $\mathrm{I}^{3}$ and STICH II, ${ }^{4}$ have shown that patients with ICH did not benefit from surgical interventions, which consist primarily of the craniotomy procedure. However, the results of other recent clinical studies have supported the effectiveness of MIPD and $\mathrm{ES}$ in $\mathrm{ICH} .{ }^{5,6}$

Both MIPD and ES are minimally invasive and have drastically reduced the manipulation of viable brain tissue during operational procedure compared with the craniotomy procedures; these have shown great advantages for the treatment of ICH. ${ }^{5,6}$ However, the indication of MIPD, or ES specified, has not been clear until recently. MIPD and ES are quite different in details, while few studies have compared the curative effect of MIPD and ES for ICH patients. It is difficult to determine which one of these is the optimal choice for an individualized ICH patient. In the present study, we conducted a retrospective study of 112 patients with ICH in basal ganglia to analyze the curative effect of MIPD and ES.

\section{Methods}

\section{Data sources}

On a retrospective basis, clinical data of 112 patients were evaluated, who had been diagnosed with spontaneous ICH between October 2013 and October 2014. The study was approved by the Ethics Committee of Tangdu Hospital, The Fourth Military Medical University (Tangdu-2015089). Written informed consent was obtained from all patients whose medical files were used in the present research. All patients were admitted to Tangdu hospital within 24 hours after disease onset. The diagnosis of ICH was confirmed by CT imaging of the head and was made according to the diagnostic criteria of guidelines for the primary prevention of stroke. ${ }^{7}$ The patients were divided into the following two groups: MIPD group and ES group.

Inclusion criteria for patients in this study were as follows: 1) meeting the diagnostic criteria of spontaneous $\mathrm{ICH} ;{ }^{7}$ 2) CT: hematoma in the basal ganglia, with (or without) the brain ventricle; 3) $\mathrm{HV} \geq 30 \mathrm{~mL}$; 4) preoperative GCS score $\geq 4 ; 5$ ) hospital admission within 24 hours of onset of symptoms; 6) age $\leq 75$ years; and 7 ) no serious coagulation disorders.

Exclusion criteria were 1) cerebral hemorrhage induced by intracranial aneurysms, arteriovenous malformations, tumors, infarction, or trauma; 2) coagulopathy, including thrombocytopenia and hepatitis; 3) severe cardiac, hepatic, renal, or pulmonary dysfunctions; 4) prior history of stroke with neurological deficits; 5) subtentorial hemorrhages; and 6) untreated and discharged patients or patient death after admission.

\section{Definition of baseline index}

ICH was diagnosed using a CT scan (slice thickness: $4.80 \mathrm{~mm}$ ), with the hematoma located in the basal ganglia (internal and/or external capsule, caudate nucleus, putamen, or more than one of the abovementioned structures). HV was calculated using MATLAB R2012b (Math Work, Natick, MA, USA) semiautomatically. The presence of IVH was recorded, but IVH was not included in the calculation of ICH volume. The ICH score of each patient was calculated according to the method described by Hemphill et al. ${ }^{8}$

Blood pressure was measured repeatedly after patients were admitted to hospital. Judgment of DM was based on the history of DM or anti-DM medications. The GCS scores were assessed on admission and classified into two categories: mild (9-14) and severe (4-8).

\section{Interventions}

\section{Basal treatment}

All patients received medical management according to the guidelines of the American Heart Association/American Stroke Association Stroke Council for the treatment of spontaneous $\mathrm{ICH}$ in adults, ${ }^{9}$ including treatments for the control of blood pressure, cerebral edema, and glycemia; gastric protection; nutritional support; and the prevention of complications.

\section{MIPD}

All operations were performed under general anesthesia. The procedure was similar to the method described by Mould et al. ${ }^{10}$ Using the stereotactic frame (Gaoxun Company, Guangzhou, Guangdong, China), a 10-French cannula was placed by stereotactic means into the hemorrhage clot according to preoperative CT, with the tip at two-thirds the length of the long axis. Special attention was paid to avoid important cortical function areas, as well as to avoid main blood vessels. After confirming the positioning of a cannula with postoperative CT, intraclot administration of urokinase (1.0×104 IU; Ndpharm, Nanjing, Jiangsu, China) was performed every 8 hours, up to nine doses, or until the residue hematoma was $<10 \mathrm{~mL}$. CT scans were obtained every 24 hours to evaluate drainage, or as clinically indicated. 


\section{ES}

ES procedures were based on the method described by Kuo et al. ${ }^{11}$ Transtemporal or transfrontal approach was selected when the route provided the shortest distance between the cortical surface and the hematoma on the preoperative CT scan. A transparent plastic sheath $(10 \mathrm{~mm}$ in outer diameter; Weike Company, Changzhou, Jiangsu, China) was inserted into the clot via a bur hole in the skull. A $4 \mathrm{~mm} 0^{\circ}$ rod lens endoscope (HOPKINS II; Karl Storz Company, Tuttlingen, Germany) was introduced into the sheath to provide illumination and visualization during hematoma evacuation. All of the blood clot that could be observed during an operation was evacuated using an aspirator $(20 \mathrm{~cm}$ in length, $1.5 \mathrm{~mm}$ in inner diameter, and $2.0 \mathrm{~mm}$ in outer diameter; Hujiang Company, Shanghai, China). Hemorrhagic spots were coagulated using a bipolar coagulator.

\section{Evaluation of curative effect}

The following parameters were collected to evaluate curative effect: 1) for short-term outcomes, several parameters were included, such as ER at days 1 and 3, PHE, rebleeding, residue hematoma, anesthetic time, blood loss in operation, common complications, and total expenditure. The ER was calculated using the formula: (preoperative $\mathrm{HV}$ - residue $\mathrm{HV}$ )/preoperative HV. The volume of PHE was measured using MATLAB R2012b semiautomatically, and 2) for long-term outcomes, the primary outcome was functional independence at 1 year using the GOS, BI, and mRS. The cumulative case fatality was also recorded.

\section{Statistical analysis}

All statistical analyses were done using SPSS 19.0 software package (SPSS Inc., Chicago, IL, USA). Values are expressed as mean \pm SD. Quantitative data were analyzed by the two-sample $t$-test, while categorical data were compared using chi-square tests. A $P<0.05$ was considered statistically significant.

\section{Results \\ General results}

Baseline data are shown in Table 1. Data from a total of 112 consecutive patients with $\mathrm{ICH}$ in the basal ganglia were collected in this study. There were 54 patients enrolled in the MIPD group and 58 patients enrolled in the ES group. The mean patient age was $59.7 \pm 7.5$ years and $61.8 \pm 9.9$ years for MIPD and ES groups, respectively $(P=0.599)$. The mean HV was $63.1 \pm 15.8 \mathrm{~mL}$ for the MIPD group and $64.3 \pm 17.2 \mathrm{~mL}$ for the ES group $(P=0.703)$. Patients with IVH accounted for
Table I Clinical baseline parameters

\begin{tabular}{llll}
\hline Clinical parameters & MIPD (54) & ES (58) & P-value \\
\hline Female (n/\%) & $29 / 53.7$ & $33 / 55.9$ & 0.734 \\
Age (years) & $59.7 \pm 7.5$ & $61.8 \pm 9.9$ & 0.599 \\
Weight (kg) & $70.7 \pm 7.8$ & $71.5 \pm 7.0$ & 0.813 \\
SBP (mmHg) & $175.8 \pm 15.2$ & $172.3 \pm 18.6$ & 0.650 \\
Pre-o HV (mL) & $63.1 \pm 15.8$ & $64.3 \pm 17.2$ & 0.703 \\
$\quad 30-60 \mathrm{~mL}(\mathrm{n} / \%)$ & $29 / 53.7$ & $32 / 55.2$ & 0.876 \\
$\quad>60 \mathrm{~mL}(\mathrm{n} / \%)$ & $25 / 46.3$ & $26 / 44.8$ & \\
Direction (left/right) & $29 / 25$ & $28 / 30$ & 0.566 \\
IVH (n/\%) & $16 / 29.6$ & $18 / 31.0$ & 0.872 \\
GCS score & $8.4 \pm 2.9$ & $8.6 \pm 3.0$ & 0.801 \\
$\quad 9-14(\mathrm{n} / \%)$ & $26 / 48.1$ & $28 / 48.3$ & 0.989 \\
$\quad 4-8(\mathrm{n} / \%)$ & $28 / 51.9$ & $30 / 51.7$ & \\
ICH score & $2.31 \pm 0.505$ & $2.25 \pm 0.512$ & 0.566 \\
Diabetes (n/\%) & $7 / 12.9$ & $10 / 17.2$ & 0.528 \\
\hline
\end{tabular}

Abbreviations: MIPD, minimally invasive puncture and drainage; ES, endoscopic surgery; SBP, systolic blood pressure; Pre-o, preoperative; HV, hematoma volume; $\mathrm{IVH}$, intraventricular hemorrhage; GCS, Glasgow Coma Scale; ICH, intracerebral hemorrhage.

$29.6 \%$ and $31.0 \%$ of the MIPD and ES groups, respectively $(P=0.872)$. There were no significant differences between the two groups among other baseline data, including gender, ICH score, age, body weight, blood pressure, initial GCS score, and DM.

\section{Short-term outcomes}

Table 2 shows the short-term outcomes within 30 days after ictus. The ER on postoperative day 1 in the ES group (82.7 \pm 23.7$)$ was significantly higher than that in the MIPD group (41.2 $\pm 20.0, P=0.001)$. After thrombolytic treatment with urokinase, the ER between MIPD and ES groups was not significantly different on postoperative day $3(P=0.886)$.

Table 2 Short-term outcomes

\begin{tabular}{|c|c|c|c|}
\hline Clinical outcomes & MIPD (54) & ES (58) & P-value \\
\hline ER at day I (\%) & $41.2 \pm 20.0$ & $82.7 \pm 23.7$ & 0.001 \\
\hline ER at day $3(\%)$ & $86.2 \pm 10.3$ & $85.5 \pm I I .3$ & 0.886 \\
\hline Residue hematoma (mL) & $9.0 \pm 2.8$ & $8.6 \pm 2.5$ & 0.740 \\
\hline PHE at day 7 (mL) & $64.3 \pm 14.2$ & $77.2 \pm 8.5$ & 0.024 \\
\hline Rebleeding (n/\%) & $2 / 3.7$ & $3 / 5.1$ & 0.707 \\
\hline Anesthetic time (minutes) & $50.9 \pm 5.6$ & $90.9 \pm 10.0$ & $<0.001$ \\
\hline Blood loss (mL) & $26.3 \pm 6.8$ & $123.6 \pm 19.5$ & $<0.001$ \\
\hline Pulmonary infection (n/\%) & $2 / 3.7$ & $4 / 6.8$ & 0.453 \\
\hline Infectious meningitis ( $\mathrm{n} / \%)$ & $1 / 1.9$ & $2 / 3.4$ & 0.601 \\
\hline Hypoproteinemia (n/\%) & $4 / 7.4$ & $|4 / 24|$. & 0.016 \\
\hline Digestive tract ulcer (n/\%) & $5 / 9.3$ & |4/24.| & 0.036 \\
\hline Renal failure (n/\%) & $2 / 3.7$ & $\mathrm{I} / \mathrm{I} .7$ & 0.517 \\
\hline Epilepsy (n/\%) & $2 / 3.7$ & $3 / 5.1$ & 0.707 \\
\hline Hospitalization length (day) & $9.6 \pm 2.8$ & $10.4 \pm 3.1$ & 0.549 \\
\hline Total expenditure & $39.3 \pm 3.6$ & $45.2 \pm 4.7$ & 0.006 \\
\hline ( $¥$, thousand yuan) & & & \\
\hline
\end{tabular}

Abbreviations: MIPD, minimally invasive puncture and drainage; ES, endoscopic surgery; ER, evacuation rate; $\mathrm{PHE}$, perihematoma edema. 
Volume of residue hematoma was calculated after removal of the intracerebral cannula. There was no significant difference in residue HV between the MIPD and ES groups $(P=0.740$, Figures 1 and 2 for individual patient of MIPD and ES groups, respectively). PHE was examined on day 7 after ictus. PHE of the MIPD group (64.3 \pm 14.2$)$ was smaller than that of the ES group (77.2 $\pm 8.5, P=0.024$; Figures $1 \mathrm{C}$ and $2 \mathrm{C}$ ). It was not possible to stop bleeding during the MIPD operation. However, the rebleeding rate of the MIPD group was not significantly higher than that of the ES group $(P=0.707)$. The anesthetic time and blood loss of the MIPD group were significantly lower than those of the ES group $(P<0.001)$. In addition, the incidence of hypoproteinemia $(P=0.016)$ and digestive tract ulcer $(P=0.036)$ was also lower in the MIPD group than in the ES group. Incidence of other complications, such as infectious meningitis, renal failure, and epilepsy, was indistinguishable between the groups. There was no significant difference in length of hospitalization between the groups, while the total expenditure was higher for the ES group than for the MIPD group $(P=0.006)$.

\section{Long-term outcomes}

Table 3 shows the long-term outcomes at 1 year after ictus. The cumulative total mortality was $23.2 \%(26 / 112)$, and the difference between the MIPD and ES groups was insignificant $(22.2 \%$ vs $24.1 \%, P=0.810)$. BI and $\mathrm{mRS}$ were collected from surviving patients. Analysis with regard to patients with GCS score 9-14 showed that the GOS score and BI were significantly higher $(P=0.030, P=0.040)$ in the MIPD group than in the ES group. The $\mathrm{mRS}$ was significantly lower in the MIPD group than in the ES group $(P=0.023)$. For patients with HV 30-60, the GOS score and BI were significantly higher $(P=0.023, P=0.046)$ in the MIPD group than in the ES group. The mRS was lower $(P=0.034)$ in the MIPD group than in the ES group. Other stratified variables showed no significant differences in GOS score, $\mathrm{BI}$, or mRS between groups for patients with GCS score 4-8 or $\mathrm{HV}>60$.

\section{Discussion}

Brain damage induced by $\mathrm{ICH}$ can be divided into primary injury and secondary injury. ${ }^{12}$ Primary injury is mainly the result of mechanical damage associated with the mass effect. ${ }^{13}$ Mass effect is related to the volume of the given hematoma. In the present study, both MIPD and ES techniques evacuated the hematoma effectively. On postoperative day 1, the ER of ES was significantly higher than that of MIPD. After using urokinase to dissolve residue hematoma, the ER of MIPD
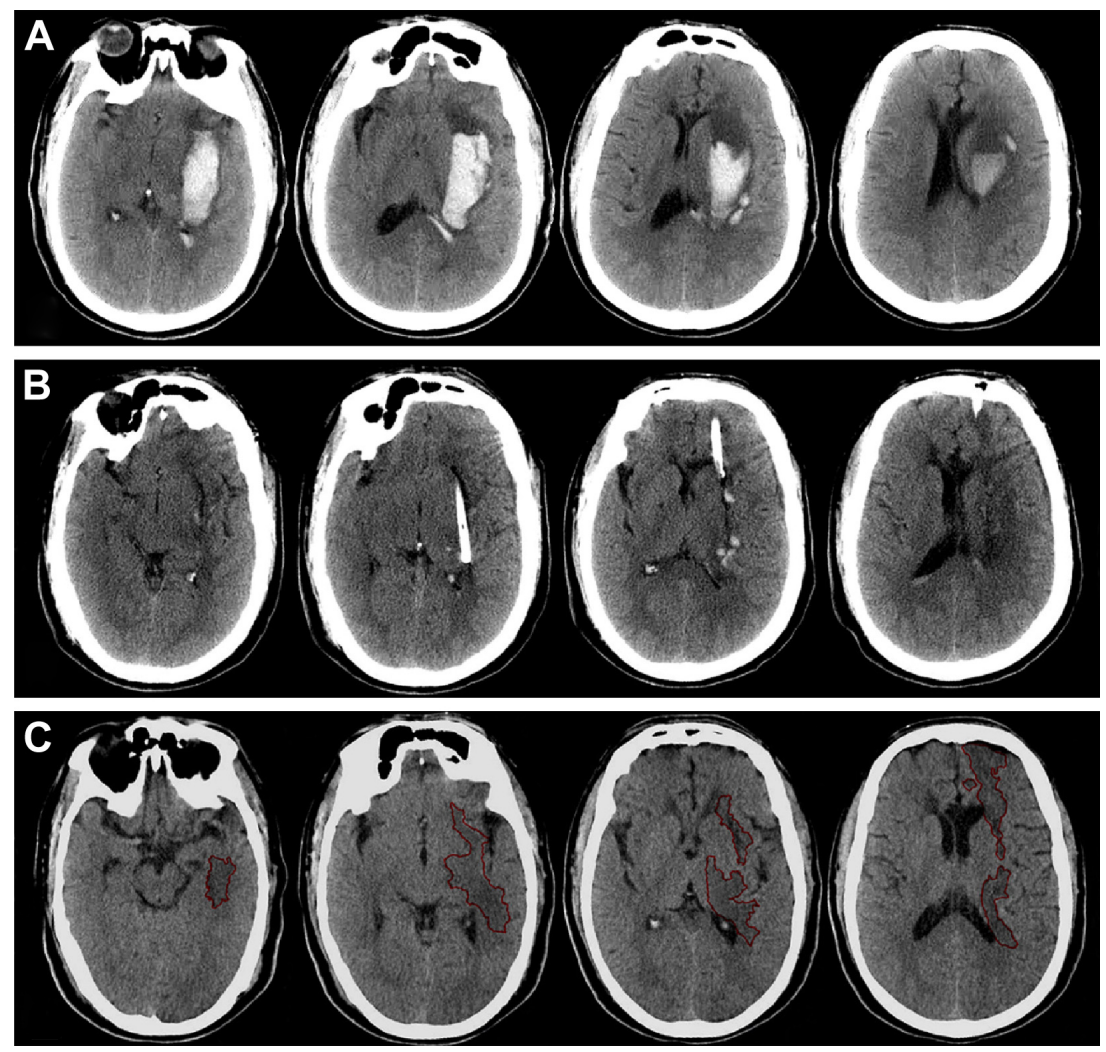

Figure I Computed tomography scan before operation (A), at postoperative day 2 (B), and postictus day 7 (C) for a minimal invasive puncture and drainage patient. Note: The perihematomal edema was semiautomatically threshold-based segmented and outlined with red line (C). 

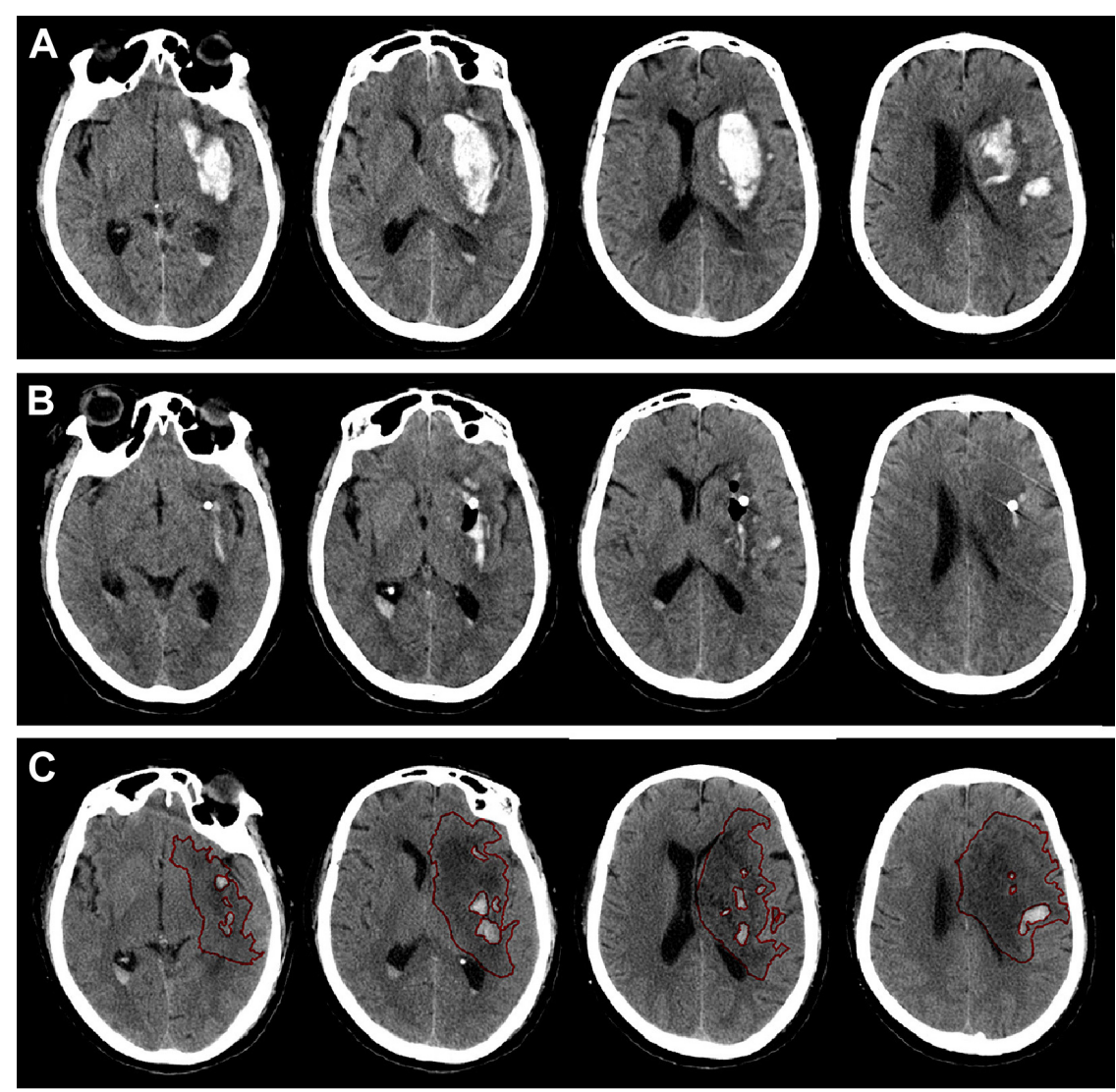

Figure 2 Computed tomography scan before operation (A), at postoperative day I (B), and postictus day 7 (C) for an endoscopic surgery patient. Note: The perihematomal edema was semiautomatically threshold-based segmented and outlined with red line (C).

did not differ significantly from that of ES on postoperative day 3. These results indicate that, although ES and MIPD had similar hematoma ERs in the acute phase, ES evacuates the hematoma more quickly than MIPD and is more potent in alleviating the mass effect of ICH.

Table 3 Long-term outcomes

\begin{tabular}{|c|c|c|c|}
\hline Clinical outcomes & MIPD & ES & $P$-value \\
\hline Case fatality (n/\%) & $12 / 22.2$ & |4/24.I & 0.810 \\
\hline \multicolumn{4}{|l|}{ GOS } \\
\hline GCS 9-14 & $4.3 \pm 0.6$ & $3.9 \pm 0.7$ & 0.030 \\
\hline GCS 4-8 & $3.5 \pm 1.0$ & $3.4 \pm 0.8$ & 0.743 \\
\hline $\mathrm{HV} 30-60 \mathrm{~mL}$ & $4.3 \pm 0.6$ & $3.8 \pm 0.9$ & 0.023 \\
\hline $\mathrm{HV}>60 \mathrm{~mL}$ & $3.3 \pm 0.8$ & $3.4 \pm 0.6$ & 0.581 \\
\hline \multicolumn{4}{|l|}{$\mathrm{BI}$} \\
\hline GCS 9-14 & $84.6 \pm 19.4$ & $77.3 \pm 13.4$ & 0.040 \\
\hline GCS 4-8 & $73.3 \pm 14.5$ & $75.3 \pm 11.4$ & 0.651 \\
\hline $\mathrm{HV} 30-60 \mathrm{~mL}$ & $83.8 \pm 10.8$ & $76.7 \pm 13.1$ & 0.046 \\
\hline $\mathrm{HV}>60 \mathrm{~mL}$ & $72.8 \pm 14.6$ & $74.8 \pm 12.1$ & 0.651 \\
\hline \multicolumn{4}{|l|}{$\mathrm{mRS}$} \\
\hline GCS 9-14 & $1.7 \pm 0.9$ & $2.3 \pm 0.7$ & 0.023 \\
\hline GCS 4-8 & $2.4 \pm 1.2$ & $2.5 \pm 1.1$ & 0.772 \\
\hline $\mathrm{HV} 30-60 \mathrm{~mL}$ & $1.9 \pm 1.1$ & $2.6 \pm 1.0$ & 0.034 \\
\hline $\mathrm{HV}>60 \mathrm{~mL}$ & $2.6 \pm 1.2$ & $2.4 \pm 1.1$ & 0.678 \\
\hline
\end{tabular}

Abbreviations: MIPD, minimally invasive puncture and drainage; ES, endoscopic surgery; GOS, Glasgow Outcome Scale; GCS, Glasgow Coma Scale; HV, hematoma volume; Bl, Barthel Index; mRS, modified Rankin Scale.
The PHE is a direct consequence of ICH (local pressure, the protein contents, and the degradation products of blood clot) and a reason for neurological deterioration after the first 24-48 hours from the onset of symptoms. ${ }^{14}$ PHE begins as soon as ICH occurs and reaches to the maximum until 3 weeks after ictus in its natural course. ${ }^{15}$ It has been reported that for hematomas with volume $<60 \mathrm{~mL}$, secondary brain injury was the dominant injury mechanism in the pathological mechanism of the ICH. ${ }^{16-18}$ Therefore, removal of the hematoma in the acute phase, via surgical techniques, is supposed to be effective in reducing PHE and to be promising toward improving the result of $\mathrm{ICH}$, especially for patients with an $\mathrm{HV}<60 \mathrm{~mL}$. The recently published result of the MISTIE II study ${ }^{10}$ revealed that MIPD treatment significantly reduced PHE volume compared with nonsurgical medical treatment. Moreover, the percentage of removed ICH was in positive correlation with the reduction of PHE, indicating that hematoma removal with MIPD technique is effective in managing PHE. According to the result of the present study, the hematoma evacuation was equally effective with both MIPD and ES, but the edema around the area of the removed hematoma was more pronounced on postoperative day 7 in the ES group: This indicates that the 
more extensive manipulations in the brain with endoscopic hematoma removal (placement and movement of working cannula in the brain, coagulation in brain tissues) result in more edema reaction.

Definitive surgical techniques for ICH have not been established until the present. In the past several decades, open craniotomy with hematoma evacuation has ever been the primary choice. ${ }^{19}$ It was characterized by good visibility, complete removal of the hematoma, easy hemostasia, and satisfactory reduction of intracranial pressure. ${ }^{19}$ However, several randomized trials in recent years have failed to show neurological improvement from surgical treatments, which are primarily open craniotomy compared with conservative medical treatment. ${ }^{3,4,20}$ Open craniotomy is accompanied with shortcomings associated with the procedure, such as long duration of surgery, mass blood loss, stretching and electrocoagulation of brain, and a potential for a series of pathophysiological changes after craniotomy. These shortcomings may offset the benefit of hematoma evacuation and ICP reduction of open craniotomy. ${ }^{3}$ The lack of clear benefit from open craniotomy has led to the significance of the use of minimally invasive techniques for the evacuation of acute ICH. Both MIPD and ES are minimally invasive techniques, while the MIPD procedure requires less invasive manipulations in brain tissue than ES. ${ }^{6,10}$ Results of the present study also showed that the MIPD group had less blood loss and shorter anesthetic time. Moreover, the incidence of hypoproteinemia and digestive tract ulcer, which reflects the systemic states of patients, was also lower in the MIPD group than in the ES group. All these results suggest that MIPD is less invasive than ES. Rebleeding is a serious complication of ICH. Careful hemostasis can be achieved by coagulations in ES, while it is impossible to stop bleeding with any tools during MIPD. However, the rebleeding rate of the MIPD group was not higher than that of the ES group, indicating that MIPD did not increase the risk of rebleeding.

In the present study, there was no significant difference in case fatality between the MIPD and ES groups. The primary outcome was functional independence at 1-year postictus. Three indexes were used to evaluate the functional outcomes: GOS, BI, and mRS. Since the HV and GCS score are independent prognostic factors for clinical outcomes of $\mathrm{ICH},{ }^{21,22}$ the functional outcomes of patients at 1 year after ictus were analyzed according to different HV and GCS scores. For patients with HV 30-60 mL or GCS score 9-14, the GOS score at 1-year postictus of the MIPD group was significantly higher than the ES group. Similarly, according to the BI and mRS, patients of the MIPD group with HV
30-60 mL or GCS score 9-14 were with better outcomes than patients of the ES group. In contrast, for patients with $\mathrm{HV}>60 \mathrm{~mL}$ or GCS score 4-8, the GOS score, BI, and mRS at 1-year postictus of the MIPD group were not significantly different from those of the ES group. Previous studies have already compared MIPD or ES with open surgery and have shown that the curative effect of MIPD or ES surpassed that of open craniotomy. ${ }^{23,24}$ MIPD and ES play more and more important roles in the treatment of $\mathrm{ICH} .{ }^{5}$ In addition, there are studies that compared the curative effect of MIPD and ES. $^{24,25}$ However, in these studies differences in surgical techniques (MIPD and ES), which may impact the clinical outcomes, were not specially discussed. These studies also lacked uniform baseline characteristics prior to operation ${ }^{25}$ or mainly focused on noncomatose patients. ${ }^{24}$ In our study, the preoperative baseline characteristics of both groups were uniform and we selected comatose as well as noncomatose patients. The results of the present study indicate that MIPD and ES have similar impact on the fatality of ICH patients, while patients with HV 30-60 mL or GCS score 9-14 may benefit more from the MIPD than the ES, compared with those with $\mathrm{HV}>60 \mathrm{~mL}$ or GCS score $4-8$, in terms of increasing neurologic functional independence.

\section{Conclusion}

Our results showed that ES evacuated hematoma more quickly than MIPD, while MIPD was less invasive than ES. MIPD may be more effective than ES for achieving a better functional independence in patients with $\mathrm{HV} 30-60 \mathrm{~mL}$ or GCS score 9-14. These preliminary results warrant further study of large, prospective, randomized trials in the near future.

\section{Disclosure}

The authors report no conflicts of interest in this work.

\section{References}

1. Dennis MS. Outcome after brain haemorrhage. Cerebrovasc Dis. 2003; 16(suppl 1):9-13.

2. Russell MW, Boulanger L, Joshi AV, Neumann PJ, Menzin J. The economic burden of intracerebral hemorrhage: evidence from managed care. Manag Care Interface. 2006;19(6):24-28, 34.

3. Mendelow AD, Gregson BA, Fernandes HM, et al; STICH investigators. Early surgery versus initial conservative treatment in patients with spontaneous supratentorial intracerebral haematomas in the international surgical trial in intracerebral haemorrhage (STICH): a randomised trial. Lancet. 2005;365(9457):387-397.

4. Mendelow AD, Gregson BA, Rowan EN, et al; STICH II Investigators. Early surgery versus initial conservative treatment in patients with spontaneous supratentorial lobar intracerebral haematomas (STICH II): a randomised trial. Lancet. 2013;382(9890):397-408. 
5. Barnes B, Hanley DF, Carhuapoma JR. Minimally invasive surgery for intracerebral haemorrhage. Curr Opin Crit Care. 2014;20(2): $148-152$.

6. Beynon C, Schiebel P, Bosel J, Unterberg AW, Orakcioglu B. Minimally invasive endoscopic surgery for treatment of spontaneous intracerebral haematomas. Neurosurg Rev. 2015;38(3):421-428. Discussion 428.

7. Meschia JF, Bushnell C, Boden-Albala B, et al; American Heart Association Stroke Council; Council on Cardiovascular and Stroke Nursing; Council on Clinical Cardiology; Council on Functional Genomics and Translational Biology; Council on Hypertension. Guidelines for the primary prevention of stroke: a statement for healthcare professionals from the American Heart Association/American Stroke Association. Stroke. 2014;45(12):3754-3832.

8. Hemphill JC, Bonovich DC, Besmertis L, Manley GT, Johnston SC. The ICH score: a simple, reliable grading scale for intracerebral hemorrhage. Stroke. 2001;32(4):891-897.

9. Connolly ES Jr, Rabinstein AA, Carhuapoma JR, et al. Guidelines for the management of aneurysmal subarachnoid hemorrhage: a guideline for healthcare professionals from the American Heart Association/ American Stroke Association. Stroke. 2012;43(6):1711-1737.

10. Mould WA, Carhuapoma JR, Muschelli J, et al; MISTIE Investigators. Minimally invasive surgery plus recombinant tissue-type plasminogen activator for intracerebral hemorrhage evacuation decreases perihematomal edema. Stroke. 2013;44(3):627-634.

11. Kuo LT, Chen CM, Li CH, et al. Early endoscope-assisted hematoma evacuation in patients with supratentorial intracerebral hemorrhage: case selection, surgical technique, and long-term results. Neurosurg Focus. 2011;30(4):E9.

12. Xi G, Keep RF, Hoff JT. Mechanisms of brain injury after intracerebral haemorrhage. Lancet Neurol. 2006;5(1):53-63.

13. Qureshi AI, Mendelow AD, Hanley DF. Intracerebral haemorrhage. Lancet. 2009;373(9675):1632-1644.

14. Sangha N, Gonzales NR. Treatment targets in intracerebral hemorrhage. Neurotherapeutics. 2011;8(3):374-387.
15. Staykov D, Wagner I, Volbers B, et al. Natural course of perihemorrhagic edema after intracerebral hemorrhage. Stroke. 2011;42(9):2625-2629.

16. Rabinstein AA, Atkinson JL, Wijdicks EF. Emergency craniotomy in patients worsening due to expanded cerebral hematoma: to what purpose? Neurology. 2002;58(9):1367-1372.

17. Huang FP, Xi G, Keep RF, Hua Y, Nemoianu A, Hoff JT. Brain edema after experimental intracerebral hemorrhage: role of hemoglobin degradation products. J Neurosurg. 2002;96(2):287-293.

18. Georgiadis $\mathrm{P}, \mathrm{Xu} \mathrm{H}$, Chua $\mathrm{C}$, et al. Characterization of acute brain injuries and neurobehavioral profiles in a rabbit model of germinal matrix hemorrhage. Stroke. 2008;39(12):3378-3388.

19. Bosel J, Zweckberger K, Hacke W. Haemorrhage and hemicraniectomy: refining surgery for stroke. Curr Opin Neurol. 2015;28(1):16-22.

20. Zuccarello M, Brott T, Derex L, et al. Early surgical treatment for supratentorial intracerebral hemorrhage: a randomized feasibility study. Stroke. 1999;30(9):1833-1839.

21. Mitra D, Das SK, Ganguly PK, Roy TN, Maity B, Munshi AK. Prognostic factors in intracerebral haemorrhage. J Assoc Physicians India. 1995; 43(9):602-604.

22. Poon MT, Fonville AF, Al-Shahi Salman R. Long-term prognosis after intracerebral haemorrhage: systematic review and meta-analysis. J Neurol Neurosurg Psychiatry. 2014;85(6):660-667.

23. Hill MD, Silver FL, Austin PC, Tu JV. Rate of stroke recurrence in patients with primary intracerebral hemorrhage. Stroke. 2000;31(1):123-127.

24. Cho DY, Chen CC, Chang CS, Lee WY, Tso M. Endoscopic surgery for spontaneous basal ganglia hemorrhage: comparing endoscopic surgery, stereotactic aspiration, and craniotomy in noncomatose patients. Surg Neurol. 2006;65(6):547-555. [discussion 555-546].

25. Nishihara T, Morita A, Teraoka A, Kirino T. Endoscopy-guided removal of spontaneous intracerebral hemorrhage: comparison with computer tomography-guided stereotactic evacuation. Childs Nerv Syst. 2007; 23(6):677-683.
Neuropsychiatric Disease and Treatment

\section{Publish your work in this journal}

Neuropsychiatric Disease and Treatment is an international, peerreviewed journal of clinical therapeutics and pharmacology focusing on concise rapid reporting of clinical or pre-clinical studies on a range of neuropsychiatric and neurological disorders. This journal is indexed on PubMed Central, the 'PsycINFO' database and CAS,

\section{Dovepress}

and is the official journal of The International Neuropsychiatric Association (INA). The manuscript management system is completely online and includes a very quick and fair peer-review system, which is all easy to use. Visit http://www.dovepress.com/testimonials.php to read real quotes from published authors. 